\title{
TOTAL CORPUS CALLOSUM VOLUME IN PATIENTS WITH SYSTEMIC LUPUS ERYTHEMATOSUS ACCORDING TO AGE AT DISEASE ONSET - A LONGITUDINAL STUDY
}

Paulo Rogério Julio ${ }^{1}$, Renan Bazuco Frittoli ${ }^{1}$, Roberto Marini ${ }^{1}$, Lilian Tereza Lavras Costallat ${ }^{1}$, Leticia Rittner ${ }^{1}$, Simone Appenzeller ${ }^{1}$,

1.Universidade Estadual de Campinas, Campinas (SP), Brazil.

*Corresponding author: appenzellersimone@gmail.com

\section{BACKGROUND}

The neuropsychiatric manifestations (NP) associated with systemic lupus erythematosus (SLE) include alterations in the central and peripheral nervous system. Atrophy of the corpus callosum (CC) may be directly associated with the presence of these manifestations.

\section{METHODS}

According to the ACR criteria, 68 patients with cSLE with a mean age of 27.6 years $(S D=4.4)$ and disease duration of 15.0 years $(\mathrm{SD}=4.8)$ and two control groups consisting of 44 patients with aSLE with mean age of $36.2(\mathrm{SD}=3.7)$ and disease duration of 14.1 years $(S D=4.2)$ and 58 healthy controls with a mean age of 31.8 years $(S D=5.4)$ were included. All patients and healthy controls underwent two magnetic resonance imaging (MRI) scans (interval 13.3 months [SD $=8.56]$ ). The MRI images were obtained with a 3-tesla machine, with coronal, sagittal and axial acquisitions. All participants underwent neurological and psychiatric evaluation during the MRI exams. The analysis of the corpus callosum was performed by automated segmentation method. Relevant data was collected by reviewing medical records (clinical changes, medications in use, disease activity and damage rate, laboratory tests and autoantibody research).

\section{RESULTS}

At study entry, we found a statistical difference in the assessment of the total volume of the CC between CSLE and HC (3158.7; SD \pm 579.9 vs. 3600.3; SD \pm 531.6 [p < 0.001]), and between aSLE and HC (3214.6; SD \pm 562.9 vs. 3600.3; SD \pm 531.6 [p 0.001]). Between cSLE and aSLE there was no difference. In the second RMI, we found a statistical difference in the assessment of the total CC volume between cSLE and aSLE (2708.0; SD \pm 451.1 vs. 3042.6; SD \pm 639.8 [p 0.007]), between cSLE and HC (2708.0; $\mathrm{SD} \pm 451.1$ vs. 3296.3; SD \pm 455.1 [p 0.002]), between aSLE and HC there was no statistically significant difference. In the comparison between RMRI one and two, we found a statistically significant difference (3158.7; SD \pm 579.9 vs. 2708.0.3; SD \pm 455.1 $[p<0.001]$ ) in he cSLE group. No difference between follow-up MRI of aSLE and HC groups was observed.

\section{CONCLUSION}

We observed a decrease in CC volume in patients with CSLE when compared to $\mathrm{HC}$ and patients with aSLE with similar disease duration. The progression of atrophy could explain worse prognosis of NP involvement in cSLE.

\section{KEYWORDS}

Corpus callosum, Systemic lupus erythematosus, Neuropsychiatric manifestations. 